\title{
Broncho-vaxom alleviates persistent allergic rhinitis in patients by improving Th1/Th2 cytokine balance of nasal
} mucosa*

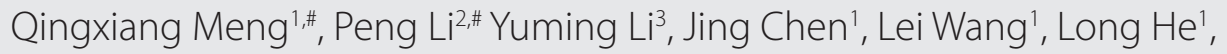 \\ Jinghua Xie' ${ }^{1}$ Xionghui Gao ${ }^{1}$ \\ Rhinology 57: 6, $451-459,2019$ \\ https://doi.org/10.4193/Rhin19.161
}

' Department of Otorhinolaryngology Head and Neck Surgery, Guangzhou First People's Hospital, School of Medicine, South China *Received for publication:

University of Technology, Guangzhou, China

April 17, 2019

2 Department of Otorhinolarygology Head and Neck Surgery, Fifth Hospital, Guangzhou Medical University, Guangzhou, China

Accepted: June 30, 2019

${ }^{3}$ Department of Otorhinolaryngology Head and Neck Surgery, The Second People's Hospital of Shunde, Foshan, China

\#These authors contributed equally

\begin{abstract}
Background: Probiotics are mainly distributed in the mucosal system and have the ability to enhance mucosal barrier function and regulate immune responses. Broncho-Vaxom (BV), as a probiotic, has been applied to patients suffering from respiratory tract infections, but its potential effectiveness in allergic rhinitis (AR) has not been evaluated in human. This study aimed to investigate the clinical efficacy of BV in patients with persistent $A R$ and to elucidate the underlying cellular mechanisms.
\end{abstract}

Methods: Sixty patients with AR were enrolled to this study and were randomly assigned to the BV group ( $n=30$ ) and the placebo group $(n=30)$. Changes of clinical symptoms and laboratory parameters of allergic inflammation were measured at baseline visit, immediately after BV treatment, four weeks, and eight weeks after the BV treatment.

Results: After BV treatment, medication score in the BV group was significantly decreased compared with placebo group, along with a significant drop of the total nasal symptom score and the individual nasal symptom scores (itching score: $23.72 \pm 5.32 \%$; nasal rhinorrhea score: $18.59 \pm 4.83 \%$; sneezing score: $23.08 \pm 4.98 \%$ ). The levels of IL-4 and IL-13 in nasal lavage were diminished remarkably while the level of INF- $\gamma$ was markedly increased in the BV group. This rendered a significant reduction of the ratio of IL-4/INF- $\gamma$. Moreover, a decrease of eosinophils in nasal smear was observed after BV treatment. The BV-induced favorable changes sustained for at least four to eight weeks post BV treatment.

Conclusion: Oral administration of BV offers remarkable and sustained efficacy in alleviating AR symptoms and may be considered as an alternative therapeutic strategy for patients with persistent AR. BV acts by improving the overall mucosal immunity via restoring and maintaining the normal Th1/Th2 cytokine balance as an underlying cellular/signaling mechanism.

Key words: Broncho-Vaxom, probiotics, allergic rhinitis, immunity, Th2 inflammation, Th1/Th2 ratio, nasal mucosa, cytokines

\section{Introduction}

Allergic rhinitis (AR) is a common chronic respiratory disease characterized by mucus hypersecretion and airway hyperres- ponsiveness with characteristic symptoms of sneezing, nasal pruritus, airflow obstruction, and mostly clear nasal discharge ${ }^{(1,}$

2). AR brings about negative impacts on quality of life, perfor-

BV: Broncho-Vaxom, AR: allergic rhinitis, MS: medication score, TNSS: total nasal symptom score, INSS: individual nasal symptom score, ARIA: Allergic Rhinitis and its impact on Asthma, HE: Hematoxylin and Eosin, HPFs: high power fields, IL: interleukin, IFN: interferon, URIs: upper respiratory tract infections, PMBL: polyvalent mechanical bacterial lysate, Th: T helper. 
mance and productivity at work, and significant cost to society

(2,3). The prevalence of AR has increased sharply over the past 2-3 decades worldwide ( $20 \%$ of the global population), particularly in industrialized countries (e.g. $>40 \%$ in many populations in the United States and Europe) ${ }^{(4)}$. AR is thought to be caused by lgEmediated reactions against inhaled allergens. The characteristic eosinophilic inflammation in AR is believed to be driven by type 2 helper T cell (Th2-type inflammation or Th2-type immune response), or more precisely by the reduced Th1/Th2 ratio of nasal mucosa ${ }^{(5)}$. Despite the tremendous advances in our understandings of the cellular/molecular mechanisms of this disease, the exact pathogenesis of AR remains ambiguous and the currently available medications often do not optimally control symptoms $(3,6)$.

In an attempt to explain the pathogenic basis of allergic diseases including $A R$, investigators formulated the hygiene hypothesis based on epidemiology studies ${ }^{(7,8)}$ and the microbial hypothesis as well ${ }^{(9,10)}$. According to the hygiene hypothesis, the prevalence of $A R$ is a result of changes in environmental factors, primarily the reduced exposure to microbial antigens, which is closely linked to the development of the early immune system such that early exposure to microbial antigens prevent allergic disease. On the other hand, the microbial hypothesis proposed that microbial exposure during the perinatal period is linked to the epigenetic regulation of genes involved in allergic inflammation that determines the susceptibility to allergic diseases ${ }^{(9)}$. The microbial hypothesis rests on two related yet distinct strategic considerations. The first of these two leads to the development of the intestinal flora theory that the make-up of intestinal microflora and the microbial flora in intestine contribute critically to allergic diseases through its substantial effect on mucosal immunity ${ }^{(11)}$. Based on this strategy, probiotics are believed to be of enormous benefits to allergic diseases including AR. Indeed, evidences are mounting to incriminate environmental factors and an aberrant gut microbiota with a shift of the Th1/Th2 balance towards a Th2 response as causes of allergic diseases. But reports on the clinical efficacy and usefulness of probiotics in treating AR have been controversial and conflicting ${ }^{(12,13)}$. Apparently, the better understanding of AR in research models that are more closely relevant to clinical situations and the continuous exploration for the improvement of medications for AR are urgent tasks of both clinicians and researchers.

OM-85 Broncho-Vaxom (BV) was developed for application to patients suffering from upper respiratory tract infections (URIs), presumably based upon the second strategic consideration of the microbial hypothesis ${ }^{(14-16)}$. BV is a mixture of lyophilized fractionated alkaline extracting from eight most common bacteria strains in URIs: Haemophilus influenzae, Diplococcus pneumonia, Klebsiella pneumoniae, Klebsiella ozaenae, Staphylococcus aureus, Streptococcus pyogenes, Streptococcus viridans, and Neisseria catarrhalis. It has now become a widely used low-endotoxin immune modulator that acts through regulating macrophage activities and cytokine productions, which has been documented to efficiently reduce both the frequency and the duration of URIs ${ }^{(14-18)}$. However, research focused on the potential of BV for the treatment of AR has been rather sparse. To our knowledge, the only one study in this regard has been published recently and the authors of this study elegantly demonstrated that OM-85 BV offers a simple, low-cost treatment of AR in a mouse model of ovalbumin-induced allergic inflammation in the nasal mucosa ${ }^{(19)}$. Nevertheless, whether this result from mice can be extrapolated to human remained yet to be assessed.

The present study was designed to shed light on this issue with two specific objectives: 1) to clarify whether BV possesses any anti-AR effects in patients with persistent $A R$ and 2) to gain insight into the potential mechanisms underlying the actions of BV in human. The results from our combined clinical and experimental studies provided strong evidence for the usefulness of BV in the management of AR and a shift of the Th1/Th2 balance towards a Th1 response as a mechanism accounting for the benefits of BV.

\section{Materials and methods}

\section{Patients}

Patients with moderate to severe perennial AR were recruited to our studies, and the diagnosis of moderate to severe perennial AR was based on the clinical criteria conforming to the guideline of Allergic Rhinitis and its impact on Asthma (ARIA) ${ }^{(20)}$. Inclusion criteria included: 1) patients with moderate to severe perennial AR for $>2$ years, and 2) hypersensivity to inhaled allergen verified by CAP-Pharmacia score >Class 2 (Phadia, Uppsala, Sweden). Exclusion criteria were: 1 ) having systemic immunologic disorders, 2) subjects with intercurrent oral corticosteroid treatment and/ or $\beta$-blockers for the past 6 months, and 3 ) having received vaccination 3 months prior to this study, 4) subjects have received allergen specific immunotherapy for allergic disease before this study.

Outpatients suffering from AR were screened and enrolled. All subjects underwent a 4-week run-in observation in order to establish their baseline clinical evaluations. After 8-week screening and 4-week run-in, the patients were evaluated for eligibility prior to double-blind randomization into two groups with equal numbers: the Broncho-Vaxom (BV) group $(n=30)$ and the placebo group $(n=30)$. The patients were randomized according to the birth date (decade of month). Loratadine was allowed control the symptoms of AR. Nine patients had mild asthma and were maintained on low doses of inhaled steroids and bronchodilators as needed (Table 1 ). The medication score was assigned on a scale of $0 \sim 3$ ( $0=$ no medication use; $1=$ medication use one to three days within one week; $2=$ medication use four to six days within one week; $3=$ medication use every day within one week). In order to avoid the interference of medication adminis- 
Table 1. Clinical characteristics of patients at the baseline visit.

\begin{tabular}{|c|c|c|c|}
\hline Characteristics & BV treatment & Placebo & P-value \\
\hline Gender (male/female) & $11 / 17$ & $14 / 12$ & NS \\
\hline Ages (years) & $33.34 \pm 3.21$ & $29.33 \pm 4.13$ & NS \\
\hline TlgE (KUA/L) & $398.67 \pm 26.04$ & $411.32 \pm 37.22$ & NS \\
\hline Specific lgE D.f. (KUA/L) & $50.32 \pm 3.96$ & $55.190 \pm 5.51$ & NS \\
\hline Specific lgE D.p. (KUA/L) & $42.02 \pm 11.71$ & $46.64 \pm 7.79$ & NS \\
\hline \multicolumn{4}{|l|}{ Prick skin test (dust mite)* } \\
\hline$+\sim++$ & 10 & 14 & NS \\
\hline$+++\sim++++$ & 18 & 12 & NS \\
\hline Total nasal symptom score (TNSS) & $7.335 \pm 1.02$ & $7.62 \pm 1.12$ & NS \\
\hline \multicolumn{4}{|c|}{ Individual nasal symptom score (INSS) } \\
\hline itching score & $2.10 \pm 0.34$ & $2.01 \pm 0.16$ & NS \\
\hline nasal rhinorrhea score & $2.22 \pm 0.31$ & $2.03 \pm 0.47$ & NS \\
\hline sneezing score & $1.88 \pm 0.68$ & $2.10 \pm 0.30$ & NS \\
\hline nasal congestion score & $2.33 \pm 0.24$ & $2.42 \pm 0.21$ & NS \\
\hline Medication score (MS) & $2.51 \pm 0.38$ & $2.26 \pm 0.29$ & NS \\
\hline Patients with asthma (yes/no) & $5 / 23$ & $3 / 23$ & NS \\
\hline Patients with eczema (yes/no) & $2 / 26$ & $2 / 24$ & NS \\
\hline \multicolumn{4}{|l|}{ Inhalation allergen (RAST) } \\
\hline Dust mite & 28 & 26 & NS \\
\hline Mould & 7 & 2 & NS \\
\hline Pollen & 7 & 4 & NS \\
\hline Fur & 3 & 1 & NS \\
\hline
\end{tabular}

${ }^{*}+$ : $\mathrm{SI}<0.5 ;++: 0.5 \leq \mathrm{SI}<1.0 ;+++: 1.0 \leq \mathrm{SI}<2.0 ;++++$ : $\mathrm{SI} \geq 2.0$. SI (skin index = Allergen diameter/Histamine diameter)

tration for the evaluation of mucosal and systematic immune, patients who used oral or intranasal glucocorticoid steroids were excluded from the trial.

\section{Study protocols}

OM85-Broncho-Vaxom (BV) was purchased from OM-Pharma, Meyrin/Geneva, Switzerland. For BV administration, we followed the regimen reported by Fu et al. ${ }^{211}$. Patients in the BV group received 3-cycles of $B V$ treatment with each cycle consisting of oral administration of BV at a dosage of $7 \mathrm{mg} /$ day for consecutive 10 days followed by a 20-day resting period. The placebo group received a placebo with BV-matching shape following the same regimen as the BV group. After the baseline visit, drug treatments were initiated. Changes of clinical symptoms and laboratory parameters of allergic inflammation in nasal mucosa in all patients were measured and analyzed at the following time points: baseline visit, immediately after 3-cycles BV treatment, 4 weeks post BV treatment, and 8 weeks post BV treatment. The study protocol was approved by the Ethics Committees of the Guangzhou First People's Hospital. Written consent was obtained from each of the enrolled patients or their guardians (for patients $<18$-years old) prior to the trial. All formulations were dispensed by a pharmacist not associated with the present study. The investigators and study subjects were both blinded to the identity and the interventions.

\section{Nasal lavage fluid sample collection}

Nasal lavage fluid samples were collected at four time points as already mentioned above for our clinical observations, according to the same procedures as previously described in detail (22). Briefly, $5 \mathrm{ml}$ saline solution (pre-warmed to $37^{\circ} \mathrm{C}$ ) was sprayed into the nasal cavities. Then, the subjects were asked to lean forward and to blow the nasal fluid into a collection container. After $20 \mathrm{sec}$, the nasal lavage fluid samples were filtered to remove nasal mucus, followed by centrifugation at $4000 \mathrm{rpm}$ for $5 \mathrm{~min}$. The preparations were stored in graded test tubes and frozen at $-80^{\circ} \mathrm{C}$ until the assays.

\section{Determination of eosinophil counts in nasal smears} Nasal smears were collected from the surface of inferior turbinate by cotton swabs dampened with physiological saline as described previously in detail ${ }^{(23)}$. Briefly, the samples were 


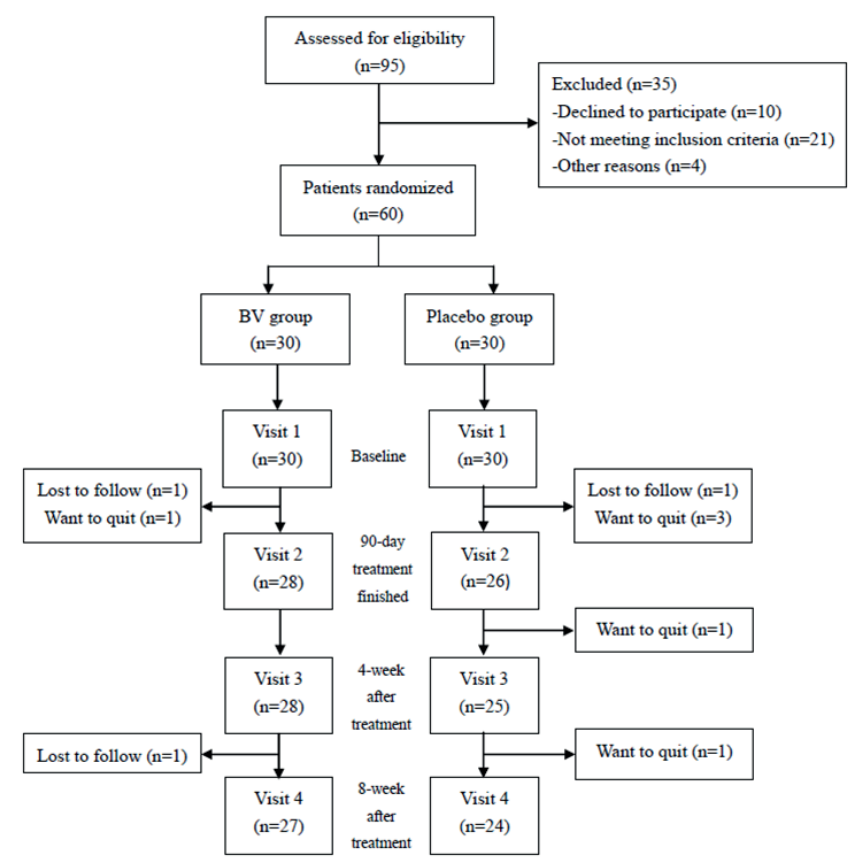

Figure 1. Study protocols.

transferred to slides by gently rolling. Following air drying, the samples were fixed with $90 \%$ ethanol and then subject to Hematoxylin and Eosin (HE) staining. A total of 100 cells per slide were evaluated for eosinophils in 5 high power fields (HPFs, magnification, 400x) under light microscope (BHS, Olympas, Japan). The mean number of eosinophils in nasal smears was determined by counting their number in randomly selected high-power fields. The counting and calculation were performed by an assigned observer in a blinded manner and in a randomized order at the end of the study.

\section{Clinical evaluation}

Clinical evaluation was carried out at four time points as described above. Patients were required to record their symptom scores and medication score. Symptom scores included the individual nasal symptom score (INSS) and the total nasal symptom score (TNSS) ${ }^{(24)}$. INSS covered the nasal rhinorrhea score, sneezing score, itching score, and congestion score separately, which were assessed on a scale of 0 to 3 ( $0=$ no symptom, $1=$ mild, $2=$ moderate, $3=$ severe). TNSS was defined as the sum of the four INSSs.

\section{Measurements of cytokines by ELISA}

The levels of interleukin (IL)-4, IL-13 and interferon (IFN)- $\gamma$ in nasal lavage were measured with ELISA-kits (IL-4, IL-13 and IFN- $\gamma$ : eBiosciences, San Diego, CA, USA) according to the manufacturer's instructions. Briefly, the nasal lavage samples were added in duplicate to 96 -well plates at $100 \mu$ per well. The appropriate biotin-conjugated antibodies included in the kits were added to
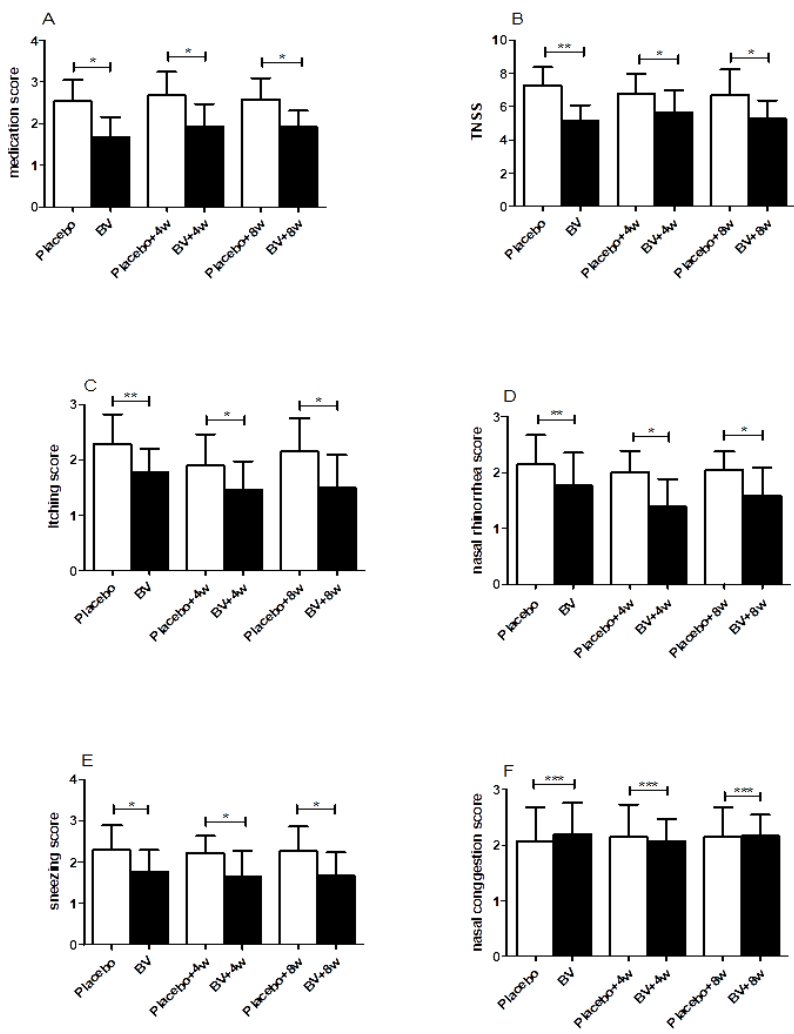

Figure 2. Change from baseline of medication and symptom scores. A: medication score of BV group significantly decreased; B: TNSS of BV group significantly decreased; C E: itching score, nasal rhinorrhea score and sneezing score of BV group significantly decreased); F: nasal congestion score did not showed significant differences compared with placebo group. $\left.{ }^{*}: \mathrm{P}<0.01{ }^{* *}: \mathrm{P}<0.05{ }^{* * *}: \mathrm{P}>0.05\right)$.

each well. The samples were incubated at room temperature for $2 \mathrm{~h}$, aspirated, and then washed 5 times. The substrate solutions were added to each well, and the reactions were incubated for $30 \mathrm{~min}$ at room temperature in the dark. Finally, the amounts of IL-4, IL-13 and INF- $\gamma$ in the nasal lavage were determined by detecting the optical density (Synergy-Ht, BIO-TEK, USA). All measurements were conducted in a blinded fashion.

\section{Serum IgE antibody assay}

Serum total $\lg E$ and specific $\lg E$ to D.p. and D.f. were determined using the Phamacia UniCAP system (ImmunoCAP, Phadia, Uppsala, Sweden).

\section{Data analysis}

The data are expressed as the mean \pm SE. Statistical analysis was carried out using SPSS13. The chi-square test or Fisher's exact test for nominal variables was used at the times of baseline assessment and each study visit between two groups. For continuous variables, the comparisons within groups were used a paired t-test for normal $\neg$ ly distributed data, or Wilcoxon's 

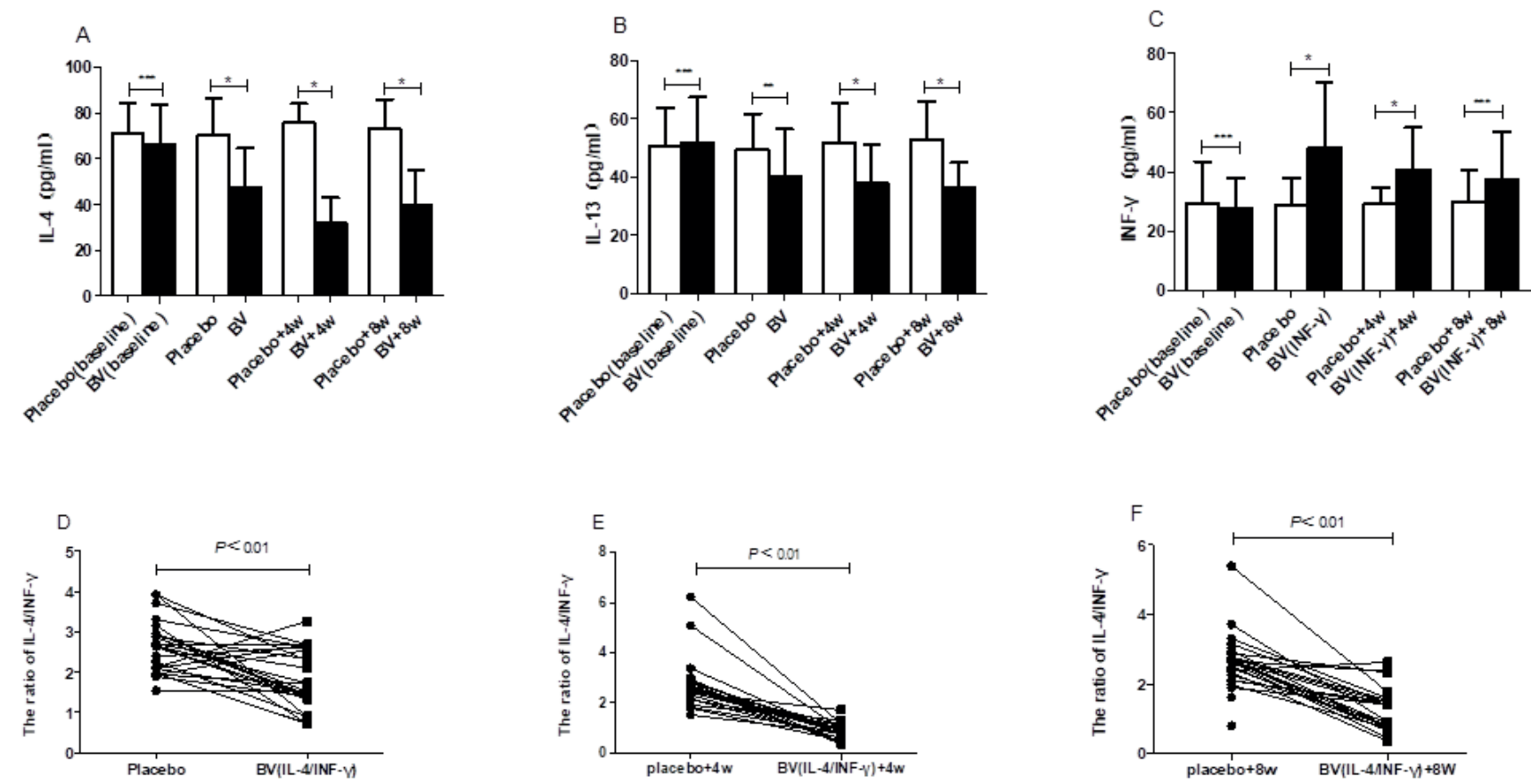

Figure 3. The expression of cytokine in nasal lavage fluid. The expression of cytokine in nasal lavage fluid. A,B: The levels of IL-4 and IL-13 in BV group significantly decreased; C: The levels of INF- $\gamma$ in BV group significantly increased; D: The ratio of IL-4/ INF- $\gamma$ in placebo group didn't show significantly change; $\mathrm{E}$ : The ratio of IL-4/ INF- $\gamma$ in BV group significantly decreased $(*: \mathrm{P}<0.01 ; * *: \mathrm{P}<0.05 ; * * *$ : $\mathrm{P}>0.05)$.

matched-pairs signed-ranks test if the data were not normally distributed; the comparisons between groups were used independent-sample Student's t-test for normally distributed data, or the Mann-Whitney U-test for non-normally distributed data. . All calculated $P$ values were 2-tailed. The differences were considered significant at $\mathrm{P}<0.05$.

\section{Results}

Clinical characteristics of the study population A total of 95 patients were screened in the Outpatient Clinics of the Department of Otolaryngology at our hospital and 60 patients were recruited to this study. The enrolled patients were randomized equally into the BV group and the placebo group. Nine patients dropped out from our studies, of which three belonged to the BV group and six to the placebo group. Thus, 27 BV and 24 placebo patients completed the present study (Figure 1) and were included in data analyses. Table 1 shows the clinical and demographic characteristics of the patients enrolled in our studies. There were no significant differences in the symptom scores and the medication score between the test patients (BV) and control subjects (placebo) at the baseline visit nor were there any age and gender differences between the two groups (Table 1).

\section{Anti-AR efficacy of BV}

After 3-cycles treatment, the overall symptoms of AR in BV patients were in general significantly relieved relative to those in the placebo control subjects (Figure 2). 1) The medication score of the BV group was markedly decreased (by $37.18 \pm 4.46 \%$; $P<0.05$; Figure $2 \mathrm{~A}$ ) compared with the placebo group. 2) TNSS was considerably lower in BV than in placebo with a $28.33 \pm 2.08 \%$ reduction $(\mathrm{P}<0.05$; Figure $2 \mathrm{~B})$. 3) For INSS, the itching score was reduced by $23.72 \pm 5.32 \%$ from $2.11 \pm 0.50$ in the placebo group to $1.65 \pm 0.49$ in the BV group $(P<0.05$; Figure $2 \mathrm{C})$. Similarly, the nasal rhinorrhea score was reduced by $18.59 \pm 4.83 \%(P<0.05$; Figure $2 \mathrm{D})$ and the sneezing score by $23.08 \pm 4.98 \%$ by BV $(P<0.05$; Figure $2 \mathrm{E})$. By comparison, the difference in the nasal congestion score did not reach a statistically significant level $(P>0.05$; Figure $2 \mathrm{~F})$.

Strikingly, the improvement of the clinical symptoms and the reduction of medication score afforded by $\mathrm{BV}$ were lasted and even further improved during the next 2-month period after the cessation of BV treatment. For example, at 4-weeks and 8-weeks post BV treatment, medication score and TNSS were remained significantly lower in $B V$ patients than in control ones $(P<0.05$; Figures $2 A$ and $B)$. Similarly, the itching and sneezing scores were maintained at lower levels comparable to the values measured right after 3-cycles BV treatment. The nasal rhinorrhea score was remained decreased for 4 weeks after BV treatment $(\mathrm{P}<0.05$; Figures $2 \mathrm{C}-2 \mathrm{E})$. The nasal congestion score, however, did not show any significant differences between the two groups ( $P>0.05$; Figure $2 F$ ). There were three adverse events in forms of slight abdominal pain been reported in the BV group. All of the adverse events were spontaneously alleviated without 


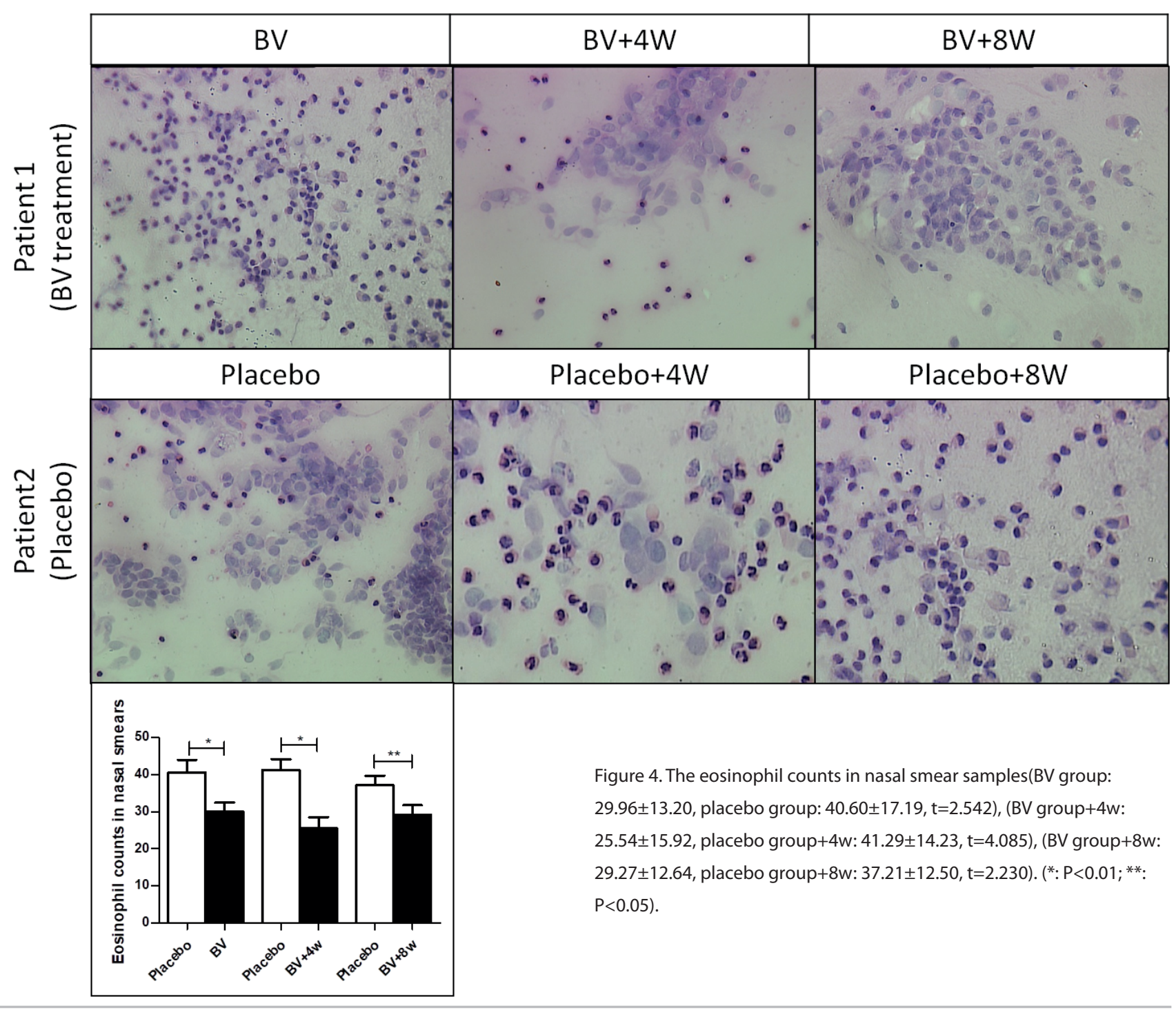

drug treatment.

\section{Effects of BV on Th1/Th2 cytokine balance}

It is known that allergic disorders are associated with a shift of the Th1/Th2 cytokine balance towards the activation of Th2 cytokines. The releases of IL-4 and IL-13 as well as IgE production act as critical cellular and molecular mechanisms ${ }^{(25,26)}$. In order to investigate if the beneficial actions of BV on AR in our studies could be explained by the favorable shift of the Th1/Th2 cytokine balance, we evaluated the effects of BV on the levels of cytokines in nasal lavage. As illustrated in Figures $3 \mathrm{~A}$ and $3 \mathrm{~B}$, the levels of both IL- 4 and IL-13 were substantially diminished by $30.69 \pm 10.52 \%$ and $20.45 \pm 14.99 \%$ after BV treatment over the values from the placebo group $(\mathrm{P}<0.05)$. In sharp contrast, the level of INF- $\gamma$, a Th1-type cytokine, was found robustly elevated in the BV group relative to the placebo group $(72.02 \pm 15.75 \%$, $\mathrm{P}<0.01$; Figure $3 \mathrm{C})$. Consequently, the ratio of IL-4/INF- $\gamma$ was markedly reduced by $B V(P<0.01$; Figure $3 D)$, indicating a shift of Th1/Th2 balance to Th1 response.
Consistent with the sustainable beneficial effects of BV on the clinical symptoms of $A R$, the favorable changes of cytokine being released in nasal lavage by $B V$ treatment also lasted for at least 8 weeks post BV treatment. The results are depicted in Figure 3: IL-4 and IL-13 were still downregulated 4-weeks and 8-weeks after BV treatment, whereas INF- $\gamma$ was still upregulated at 4 weeks post BV treatment. At 8 weeks after BV treatment, INF- $\gamma$ was still rising in its level but failed to achieve statistical significance (Figure 3C). Consequent to these changes, the IL-4/ INF- $\gamma$ ratio exhibited a marked drop in the BV group (Figure 3D).

\section{Effect of BV on eosinophil count in nasal smears}

Eosinophilia with increased number of eosinophils in the nasal lavage fluid is a hallmark of Th2-type immune response in AR (27). We reasoned that BV improves AR by suppressing Th2-type response, and then it should be able to prevent eosinophilia. To verify this notion, we quantified the eosinophil counts in nasal smears sample. As shown in Figure 4, the BV group had remarkably reduced number of eosinophils compared with the control, 
with an average of $23.46 \pm 10.19 \%$ decrease $(P<0.05)$, when measured immediately after 3-cycles BV treatment. However, this difference was mitigated at 4- and 8-weeks post-BV treatment.

\section{Discussion}

As already stated earlier, the specific aims of the present study were two-fold: 1) to clarify whether BV possesses any anti-AR efficacy in patients with persistent $A R$ and 2) to gain insight into the potential mechanisms underlying the actions of BV in human subjects. To this end, we conducted both clinical observations and laboratory measurements that allowed us to have generated a number of novel findings pertinent to our purposes. First, the 3-cycle regimen of oral BV yielded prominent benefits in the management of persistent AR. Second, BV elicited a favorable shift of the Th1/Th2 cytokine balance toward an advantageous Th1-mediated immunity characterized by amplified IFN- $\gamma$, which is likely a crucial signaling mechanism underlying the actions of BV. Finally, also remarkable is the observation that the beneficial actions of BV lasted for a period of eight weeks following the termination of treatment. Our results therefore point to the clinical efficacy and usefulness of oral BV in anti-AR treatment and prompt the encouragement and recommendation of this regimen as a promising adjunct therapeutic strategy for human AR. To the best of our knowledge, our researches represent the first clinical effort to evaluate the applicability of BV to the treatment of AR.

The importance of respiratory tract mucosa in the pathophysiology of allergic diseases has recently received increasing attentions ${ }^{(25,28)}$. Owing to its ability to modulate mucosal immunity, BV as a bacterial immunostimulator has gained wide recognitions and applications in patients suffering from URI (13-17) as well as demonstrated some effects on asthma ${ }^{(28)}$ and acute and chronic bronchitis ${ }^{(29)}$. As far as AR is concerned, the study documented by Han et al. ${ }^{(19)}$ has clearly demonstrated the efficacy of BV in the protection against AR in mice. However, these advantageous and beneficial actions of BV have not yet been examined in human subjects. The present study was motivated by these reports and it generated the clinical datum in support the animal studies. One study evaluated the efficacy of the treatment with an immunostimulating vaccine consisting of a polyvalent mechanical bacterial lysate (PMBL) in the prophylaxis of AR in 41 patients ${ }^{(31)}$. The results of this study indicate that the bacterial lysate was effective in the reduction or in the elimination of the symptoms of AR during the treatment period in comparison to a non-immunostimulating treatment. However, this bacterial lysate is distinct from BV, and therefore our studies represent the first clinical observation on the therapeutic effectiveness of BV in the treatment of AR.

Respiratory airways constantly encounter exogenous antigens and pathogens insulting from environment. The mucosal immune homeostasis in the airways forms a barrier for effective defense. It has been postulated that the Th1 immune response in mucosa produces anti-infection effects in the respiratory tract by inducing the coordinated expression of leukocytes and vascular adhesion molecules so as to recruit inflammatory cells to the site of infection. On the other hand, the Th2 immune response induces deleterious changes countering the Th1 immune response, and Th2-dominated upper airway inflammation may lead to long-term airway adverse remodeling. It is therefore believed that the balance between Th1 and Th2 (or the Th1/Th2 balance) determines the mucosal immune homeostasis ${ }^{(32)}$. The Th1/Th2 balance can be dysregulated upon antigen/pathogen insults, leading to skewed immune responses favoring Th2 response. AR is a chronic inflammatory disease orchestrated by the Th2 immune response, or more precisely by a shift of the Th1/Th2 balance towards the Th2 response. Published data have provided the evidences for BV to affect the innate immunity by regulating the activities of macrophages and the productions of proinflammatory cytokines ${ }^{(19,21,33,34)}$. BV appears to be able to evoke diverse arrays of functions on immunity, as manifested by its ability to suppress the Th2-type cytokines (IL-4, IL-5, and IL-13) and to stimulate the Th1-type cytokines (TNF- $a$, IL-1 $\beta$, IL-6, IL-8, and IFN- $\gamma$ ). Our current knowledge about the cellular effects of BV were primarily acquired from animal studies, how BV acts in human, particularly in the setting of $A R$, remained elusive. The findings of the present study serve as strong clinical evidence in support of the view that BV produces a favorable shift of the Th1/Th2 balance towards the Th1 response by inhibiting the secretions of the Th2-type cytokines (IL-4 and IL-13) and by concomitantly stimulating the productions of the Th1-type response (IFN- $\gamma$ ) in nasal mucosa. Hence, it is highly plausible that restoring and maintaining the normal Th1/Th2 cytokine balance is one of the mechanisms underlying the efficacy of BV in fighting AR. Yet, the actions of BV are likely complex involving multiple signaling pathways, and our studies do not exclude the possible participation of other factors in conferring the anti-AR efficacy of BV.

Another important finding of the present study is that BV offered long-lasting anti-AR efficacy; the beneficial effects of BV persisted for at least 8 weeks following the cessation of the final BV treatment. How this desirable property been achieved is presently unclear and remains yet to be elucidated. One explanation might be related to the potential mechanism of actions of BV. It has become increasingly apparent that respiratory tract mucosa is of paramount importance in the pathophysiology and pathogenesis of allergic diseases ${ }^{(25,28)}$. The current concepts about upper respiratory tract mucosal immunity considered mucosal immunization as the most effective approach to induce local long-term immune memory ${ }^{(35)}$. Systemic immunity and mucosal immunity in AR are two independent processes that do not function synchronously, despite that they do interact with each other to fulfill the optimal defense against antigen/patho- 
gen insults ${ }^{(36)}$. In the present study, the suppression of Th2-type inflammation was found to occur only in nasal mucosal immunity, but not in systemic immunity according to the expression of IgE antibody in serum. Whether BV has the ability to modulate the type Th2-type immune response in systemic immunity or not, that has not achieved sufficient evidence. This property of BV that can induce local long-term immune memory might partially explain the long-lasting efficacy of BV. Nonetheless, this issue is definitely subject to future studies for verification. Whether BV could be a viable option for the management of other allergic disease, such as asthma, is worth pursuing. Beyond that, although this trial has confirmed the clinical efficacy of BV on the treatment of AR in patients sensitive to dust mite and also demonstrated the long-lasting efficacy by 8 weeks follow-up after the cessation of the final BV treatment, but there were still some limitations. First, a large sample size clinical study is needed to further validate our findings in the future. Secondly, the impact and the mechanism of BV on Th2-type immune response in patients with $A R$ is needed to be further studied.

\section{Conclusion}

Oral administration of BV offers significant and sustainable efficacy in the treatment of AR and may be an alternative therapeutic strategy for human AR. BV acts by improving overall mucosal immunity in patients with persistent $A R$ via normalizing and maintaining the optimal Th1/Th2 cytokine balance as one of the underlying cellular/signaling mechanisms.

\section{Acknowledgements}

This study obtained no third-party funding.

\section{Authorship contribution}

QM: study design, clinical study and laboratory study; PL: laboratory study; YL: data analysis; JC, LW, LH, JX: clinical study; XG: clinical study and article revision.

\section{Conflict of interest}

The authors declare no conflicts of interest for the present study.

\section{References}

1. Greiner AN, Hellings PW, Rotiroti G, Scadding GK. Allergic rhinitis. Lancet 2011;378:2112-2122

2. Padjas A, Kehar R, Aleem S, et al. Methodological rigor and reporting of clinical practice guidelines in patients with allergic rhinitis: QuGAR study. J Allergy Clin Immunol 2014;133:777-783.

3. Small M, Piercy J, Demoly P, Marsden H. Burden of illness and quality of life in patients being treated for seasonal allergic rhinitis: a cohort survey. Clin Trans Allergy 2013;3:33.

4. Salo PM, Arbes SJ Jr, Jaramillo $R$, et al. Prevalence of allergic sensitization in the United States: results from the National Health and Nutrition Examination Survey (NHANES) 2005-2006. J Allergy Clin Immunol 2014;134:350-359.

5. Rosenwasser LJ. Current understanding of the pathophysiology of allergic rhinitis. Immunol Allergy Clin North Am. 2011;31:433-439

6. Gupta P, Kc T, Regmi D, et al. Diode Laser Turbinate Reduction in Allergic Rhinitis: A Cross-sectional Study. JNMA J Nepal Med Assoc $2018 ; 56(214): 949-952$.

7. Strachan DP. Family size, infection and atopy: the first decade of the "hygiene hypothesis". Thorax 2000;55(Suppl 1):S2-10.

8. Strachan DP. Hay fever, hygiene, and household size. BMJ 1989;299:1259-1260.

9. Olszak T, An D, Zeissig S, et al. Microbial exposure during early life has persistent effects on natural killer $T$ cell function. Science 2012:336:489-493.

10. Noverr MC, Huffnagle GB. The 'microflora hypothesis' of allergic diseases. Clin Exp Allergy 2005;35:1511-1520.
11. Hoh RA, Boyd SD. Gut Mucosal Antibody Responses and Implications for Food Allergy. Front Immunol 2018;9:2221.

12. Koyama T, Kirjavainen PV, Fisher C, et al. Development and pilot evaluation of a novel probiotic mixture for the management of seasonal allergic rhinitis. Can Microbiol 2010;56:730-738.

13. Helin T, Haahtela S, Haahtela T. No effect of oral treatment with an intestinal bacterial strain, Lactobacillus rhamnosus (ATCC 53103), on birch-pollen allergy: a placebo-controlled double-blind study. Allergy 2002;57:243-246.

14. Schaad UB. OM-85 BV, an immunostimulant in pediatric recurrent respiratory tract infections: a systematic review. World J Pediatr 2010;6:5-12.

15. Steurer-Stey C, Lagler L, Straub DA, Steurer J, Bachmann LM. Oral purified bacteria extracts in acute respiratory tract infections in childhood: a systematic quantitative review. Eur J Pediatr 2007;166:365-376.

16. Rozy A, Chorostowska-Wynimko J. Bacterial immunostimulants-mechanism of action and clinical application in respiratory diseases. Pneumonol Alergol Pol 2008;76:353359.

17. Razi CH, Harmanc K, Abac A,et al. The immunostimulant OM-85 BV prevents wheezing attacks in preschool children. Allergy Clin Immunol 2010;126:763-769

18. Solèr $M$, Mütterlein $R$, Cozma G, SwissGerman OM-85 Study Group. Double-blind study of OM-85 in patients with chronic bronchitis or mild chronic obstructive pulmonary disease. Respiration 2007;74:26-32.

19. Han L, Zheng CP, Sun YQ, Xu G, Wen W, Fu QL. A bacterial extract of OM-85 BronchoVaxom prevents allergic rhinitis in mice. Am
J Rhinol Allergy 2014;28:110-116.

20. Jan L. Brozek, Jean Bousquet, loana Agache, et al. Allergic Rhinitis and its impact on Asthma (ARIA) guidelines-2016 revision. J Allergy Clin Immunol 2016;140(4):950-958.

21. Fu R, Li J, Zhong H, et al. Broncho-Vaxom Attenuates Allergic Airway Inflammation by Restoring GSK3b-Related T Regulatory Cell Insufficiency. PLoS One 2014;9:e92912.

22. De Corso E, Baroni S, Romitelli F, et al. Nasal lavage CCL24 levels correlate with eosinophils trafficking and symptoms in chronic sino-nasal eosinophilic inflammation. Rhinology 2011:49:174-179.

23. Schäper C, Gustavus B, Koch B, et al. Effects of fexofenadine on infl amatory mediators in nasal lavage fluid in intermittent allergic rhinitis. J Investig Allergol Clin Immunol 2009;19:459-464.

24. Lin Z, Zhou L, Luo X,et al. Suppression of TIM-1 predicates clinical efficacy of sublingual immunotherapy for allergic rhinitis in children. Int J Pediatric Otorhinolaryngology 2013;77:1345-1349.

25. Paul WE, Zhu J. How are TH 2-type immune responses initiated and amplified? Nat Rev Immunol 2010;10:225-235.

26. Tang RB, Chen SJ. Soluble interleukin 2 receptor and interleukin 4 in sera of asthmatic children before and after a prednisoIone course. Ann Allergy Asthma Immunol 2001;86: 314e7

27. Humbles AA, Lloyd CM, McMillan SJ, et al. A critical role for eosinophils in allergic airways remodeling. Science 2004;305:17761779

28. Meng Q, Liu X, Li P, et al.The influence of house dust mite sublingual immunotherapy on the TSLP-OX40L signaling pathway in patients with allergic rhinitis. Int Forum 
Allergy Rhinol 2016;6(8):862-870.

29. Navarro S, Cossalter G, Chiavaroli C, et al. The oral administration of bacterial extracts prevents asthma via the recruitment of regulatory $\mathrm{T}$ cells to the airways. Mucosal Immunol 2011:4:53-65.

30. Orcel B, Delclaux B, Baud M, Derenne JP Oral immunization with bacterial extracts for protection against acute bronchitis in elderly institutionalized patients with chronic bronchitis. Eur Respir J 1994;7:446452.

31. Banche G, Allizond V, Mandras N, et al. Improvement of clinical response in allergic rhinitis patients treated with an oral immunostimulating bacterial lysate: in vivo immunological effects. Int J Immunopathol Pharmacol 2007;20:129-138.

32. Kim TH, Lee JY, Lee HM, et al. Remodelling of nasal mucosa in mild and severe persistent allergic rhinitis with special reference to the distribution of collagen, proteoglycans, and lymphatic vessels. Clin Exp Allergy 2010;40:1742-1754

33. Huber M, Mossmann H, Bessler WG. Th1orientated immunological properties of the bacterial extract OM-85-BV. Eur J Med Res 2005;10:209-217.

34. Luan H, Zhang Q, Wang L, Wang C, et al. OM85-BV induced the productions of IL-1 $\beta, I L-6$, and TNF- $a$ via TLR4- and TLR2mediated ERK1/2/NF-KB pathway in RAW264.7 cells. J Interferon Cytokine Res 2014;34:526-536.

35. Medina E, Guzman CA. Modulation of immune responses following antigen administration by mucosal route. FEMS Immunol Med Microbiol 2000;27:305-311.

36. Smurthwaite L, Walker SN, Wilson DR, et al. Persistent lgE synthesis in the nasal mucosa of hay fever patients. Eur J Immunol 2001; 1:3422-3431.
Qingxiang Meng, MD

Department of Otorhinolaryngology

Head and Neck Surgery

Guangzhou First People's Hospital

School of Medicine

South China University of Technology

Guangzhou

Guangdong 510810

China

Tel: $+86-20-81048990$

Fax: $+86-20-81048236$

E-mail:mqx73@163.com

ANNOUNCEMENT FELLOWSHIPS

FOUNDATION RHINOLOGY - UTRECHT

The 'Foundation Rhinology' established at Utrecht, The Netherlands

offers $\mathbf{1 0}$ Grants of $€ \mathbf{1 , 5 0 0 . 0 0}$ each

to rhinologists under the age of 36

to enable them to attend the $\mathbf{2 8 \text { th }}$ congress

of the European Rhinologic Society

in Thessaloniki, June 21-25, 2020.

\section{Requirements}

- $\quad$ Age under 36

- Specialised/working in Rhinology

\section{Applications should include:}

- Curriculum vitae with a recent photograph

- List of publications

- List of presentations at international congresses

- Letter of recommendation of Head of Department

\section{Applications may be send to:}

Prof. E.H. Huizing

Secretary 'Foundation Rhinology'

E-mail: ehhuizing@planet.nl 